\title{
Pressure Injury Prevention and Wound Management
}

\author{
Ami Hommel and Julie Santy-Tomlinson
}

The management of wounds and the prevention of pressure injuries (also known as pressure ulcers) are fundamental aspects of the management of the patient following fragility fracture, especially following hip fracture and associated surgery. Ageing skin and multiple comorbidities are significant factors in skin injury and wound healing problems. The aim of this chapter is to provide the reader with an overview of evidence-based approaches to the prevention of pressure injuries and to wound management following hip fracture surgery.

\subsection{Learning Outcomes}

At the end of the chapter, and following further study, the nurse will be able to:

- Explain the causes and pathophysiology of pressure injuries

- Recognise risk factors for pressure injury in patients following hip fracture

- Provide evidence-based care to patients at risk of pressure injury

- Discuss the factors that inhibit and enhance wound healing

- Provide evidence-based care to patients with surgical wounds following fragility fracture surgery.

\footnotetext{
A. Hommel $(\square)$

Department of Orthopaedics, Skane University Hospital, Malmö, Sweden

Department of Care Science, Malmö University, Malmö, Sweden e-mail: Ami.hommel@med.lu.se

J. Santy-Tomlinson

Faculty of Biology, Medicine and Health, Division of Nursing, Midwifery and Social Work, School of Health Sciences, The University of Manchester, Manchester, UK e-mail: Julie.santy-tomlinson@manchester.ac.uk 


\subsection{Pressure Injuries}

Pressure injuries are significant breaches of patient safety but are still relatively common following fragility fracture, especially femoral and hip fractures. The term "pressure injury" will be used as it is considered a more accurate term than "pressure ulcer" or "pressure sore" because some presentations are not open ulcers. Pressure injuries result in short- and long-term pain and distress for patients and are often considered indicators of inadequate care quality, leading to litigation. Despite the availability of evidence-based guidelines, nurses' knowledge of pressure injury prevention has been shown to be variable [1]. This is a significant factor in patients acquiring pressure injuries during hospital and care facility admissions as well as in the home care setting. An important part of the process of service improvement to reduce pressure injury incidence is to ensure that practitioners are well educated and possess the skills and knowledge of evidence-based practice in pressure injury prevention.

\subsubsection{Pathophysiology and Causes of Pressure Injuries}

Pressure injuries are localised areas of soft tissue damage that typically occur in a people who are elderly, have limited mobility or are confined to bed or chair by an acute or chronic health problem, injury or surgery and who have impaired nutrition, as is often the case for patients who are frail and have fragility fractures. These factors mean that the tolerance of the individual's skin and underlying tissues to forces that damage the skin and circulation is reduced. Tissue damage most often occurs when skin and the underlying tissues are subjected to pressure, friction and/or shear or a combination of all three. If pressure, friction or shear are prolonged, they can result in impaired blood supply and damage to skin and underlying tissues [2]. An additional factor in skin injury is moisture, usually from urinary incontinence; if urine is in contact with the skin for prolonged periods, it can lead to incontinenceassociated dermatitis (IAD), a type of irritant contact dermatitis caused by prolonged exposure of the skin to urine, faeces [3] or other fluids such as wound exudate and sweat. In combination, pressure, friction, shear and moisture (Fig. 7.1) represent a group of extrinsic factors that healthcare workers need to modify when aiming to prevent skin damage.

In addition to the extrinsic factors discussed above, patients are also vulnerable to tissue injury because of a complex interplay between a variety of intrinsic factors that affect the skin's innate ability to resist external forces-tissue tolerance (Fig. 7.1) [2]. These factors include coexisting health conditions such as those affecting the respiratory and circulatory system which result in diminished blood, oxygen and nutrition supply to the tissues. Pulmonary disease, cardiovascular disease and diabetes are common examples of such conditions. Health conditions that 
Fig. 7.1 The central causes of pressure injuries

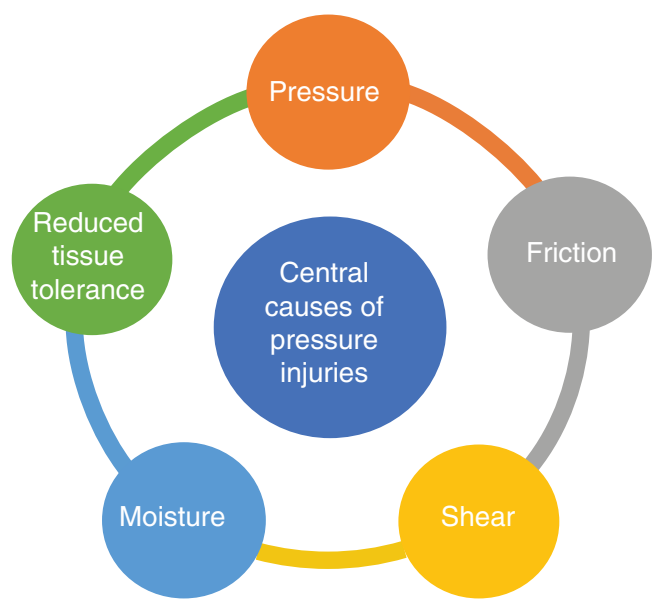

affect mobility such as osteoarthritis and neurological conditions also increase the risk of pressure injury because they restrict the patient's ability to move themselves, mobilise and change their own position in bed or chair.

\subsubsection{Classification of Pressure Injuries}

Pressure injuries are classified according to the NPUAP/EPUAP/PPIA guidance, updated in 2014 [4]. An understanding of each classification is essential in helping nurses and other staff to recognise the early development of pressure injuries so that deterioration can be prevented. The initial stage of pressure injury is usually redness of the skin, erythema, particularly over bony prominences. In the first instance, this redness indicates an area of skin that has been subjected to pressure and other forces, resulting in an inflammatory reaction that causes local dilation of blood vessels. This is called "blanching" erythema if all redness disappears when light finger pressure is applied, indicating that the local capillaries are undamaged. The patient may state that there is localised pain over a bony prominence even before erythema begins [5]. Blanching erythema is a sign that the patient's position needs to be changed as there is potential for capillary damage if pressure is not relieved. Blanchable erythema is not considered a pressure injury but an important warning sign that preventive measures are needed. If, however, the forces are not removed, blanching erythema can quickly develop into a pressure injury as indicated by category 1 in Box 7.1, non-blanchable erythema of intact skin. Each of the further categories of pressure injury indicates further tissue damage and is much more difficult to reverse than non-blanchable erythema. 


\section{Box 7.1: NPUAP/EPUAP/PPIA Classifications of Pressure Injury [4] \\ Category 1 Pressure Injury: Non-blanchable erythema of intact skin}

Intact skin with a localised area of non-blanchable erythema. This may appear differently in darkly pigmented skin. Presence of blanchable erythema or changes in sensation, temperature or firmness may precede visual changes. Colour changes do not include purple or maroon discoloration; these may indicate deep tissue injury.

Category 2 Pressure Injury: Partial-thickness loss of skin with exposed dermis

The wound bed is viable, pink or red and moist and may also present as an intact or ruptured serum-filled blister. Adipose (fat) is not visible and deeper tissues are not visible. Granulation tissue, slough and eschar are not present. These injuries commonly result from adverse microclimate and shear in the skin over the pelvis and shear in the heel. This stage should not be used to describe moisture-associated skin damage (MASD) including incontinenceassociated dermatitis (IAD), intertriginous dermatitis (ITD), medical adhesiverelated skin injury (MARS) or traumatic wounds (skin tears, burns, abrasions).

\section{Category 3 Pressure Injury: Full-thickness skin loss}

Full-thickness loss of skin, in which adipose (fat) is visible in the ulcer and granulation tissue and epibole (rolled wound edges) are often present. Slough and/or eschar may be visible. The depth of tissue damage varies by anatomical location; areas of significant adiposity can develop deep wounds. Undermining and tunnelling may occur. Fascia, muscle, tendon, ligament, cartilage and/or bone is not exposed. If slough or eschar obscures the extent of tissue loss, this is an unstageable pressure injury.

\section{Category 4 Pressure Injury: Full-thickness skin and tissue loss}

Full-thickness skin and tissue loss with exposed or directly palpable fascia, muscle, tendon, ligament, cartilage or bone in the ulcer. Slough and/or eschar may be visible. Epibole (rolled edges), undermining and/or tunnelling often occur. Depth varies by anatomical location. If slough or eschar obscures the extent of tissue loss, this is an unstageable pressure injury.

\subsubsection{Pressure Injury Prevention}

Interventions to prevent pressure injuries must be led by the latest evidence-based guidance and coordinated by the multidisciplinary team. The NPUAP/EPUAP/ PPPIA guidelines [4] provide direction for practice across the world and the following advice is based on this guidance. The implementation of guidance, evaluation of practice and regular audit and monitoring of pressure injury prevalence or incidence should be led by local experts with responsibility for service improvement [6]. This should include reporting, investigation and cause analysis of hospital- and care facility-acquired pressure injuries. 


\subsubsection{Patient Assessment}

Assessment of the patient is central to planning effective preventive care as it provides an understanding of the risk factors which can be mitigated by effective evidence-based care. The ongoing assessment of the patient should include:

- Full skin assessment as soon as possible (but within $8 \mathrm{~h}$ ) after admission and thereafter at least daily, or more frequently if the patient's health deteriorates or healthcare interventions such as procedures or surgery increase the risk of pressure injury.

- Inspection should focus on common pressure points over bony prominences such as the sacrum, buttocks, heels, the back of the head, elbows, shoulders, hips (over the greater trochanter), ischial tuberosities, sides of knees and ankles/malleoli.

- Assessment should also include taking note of any medical and other devices (e.g. casts, urinary catheters, intravenous lines, oxygen masks, straps and ties) that can lead to additional pressure points.

- Skin inspection should note any broken, discoloured, dry/flaking, papery (thin/ fragile), clammy, oedematous (puffy) or mottled skin: all of which increase the risk of, or indicate the existence of, tissue injury. Any red or discoloured skin over bony prominences indicates possible tissue damage and must be acted upon immediately to prevent deterioration.

- A structured risk assessment should be carried out as soon as possible (but within $8 \mathrm{~h}$ ) after admission to identify any risk of pressure injury development and the individual factors that require intervention. Patient characteristics that indicates potential risk of pressure injury should be documented in the risk assessment including; patient age, medical conditions impacting on tissue health and drug or other therapy impacting on tissue health. Risk assessment is not, however, an end in itself and it is important that it then leads to active intervention to modify the risk factors.

- Any existing or new pressure injuries should be recorded and classified according to the NPUAP/EPUAP/PPPIA classification system [4] in Box 7.1.

It must be stressed that assessment alone is not sufficient to reduce the incidence of pressure injuries [7] and that it is the preventive interventions that follow that are the most important actions to be taken.

\subsubsection{Evidence-Based Preventive Interventions}

Pressure injury prevention must be a priority for the whole clinical team but tends to be led by the nursing team. Pressure injuries are important indicators of the quality of nursing care and the development of an avoidable injury is a breach in patient safety. Assessment and interventions for prevention and treatment of pressure injuries need to be patient-centred. Although much research has been conducted into the causes of and most effective methods of prevention and there is much evidencebased guidance readily available, pressure injuries are still a significant problem for hospitalised patients [8]. The local, national and international incidence of hospitalacquired pressure injuries is difficult to quantify, but they are often the largest 
proportion of patient safety incidents and failures in prevention that lead to hospitalacquired pressure injuries can be viewed as healthcare-associated complications and are sometimes considered an indicator for the quality of nursing. The results of a European prevalence study, in 2002, suggested that as few as $10 \%$ of patients at risk of pressure injuries were receiving enough preventive care [9].

The UK National Health Service [10] has developed an approach to pressure injury prevention known by the acronym S.S.K.I.N. (Skin, Surface, Keep moving, Incontinence and Nutrition) which provides a useful approach to identifying evidence-based interventions for the prevention of pressure injuries as follows:

Skin: Fundamental care that helps to maintain the skin's protective purpose includes keeping the skin clean and dry using unscented skin cleansers that do not cause irritation. This is particularly important for patients with older, dry skin and for those with skin allergies and other skin conditions. It is also helpful to protect the skin's moisture barrier by regularly applying a light layer of simple, unscented moisturisers or emollients while avoiding the overuse of creams and lotions. Positioning the patient on areas of erythematous (red) skin and massaging the skin should be avoided. Massage causes friction and shear that can damage the delicate microcirculation and lead to inflammation and tissue damage.

Surface: Support surfaces on both beds and chairs should meet individual patient needs as well as operating tables during surgery. Support surface choice is based on the patient's level of mobility; those who are largely bedbound (e.g. while awaiting surgery or immediately afterwards) may benefit from the use of an alternating pressure mattress, but this should never replace the need for repositioning (see keep moving below). The relative merits of these higher specification support surfaces in preventing pressure injuries are unclear [11]. Once the patient can sit out of bed, it is essential that risk of pressure injuries is still acknowledged and a pressure redistributing cushion is used until the patient is fully mobile.

Keep moving: The aim of care should be to support remobilisation as early as possible while recognising the effects of reduced mobility during the rehabilitation phase (Chap. 6). Prolonged pressure to bony prominences and other vulnerable areas, along with friction and shear, must be avoided by regular repositioning of the patient, especially if they cannot do this for themselves or mobility is restricted. Good manual handling practice is essential in avoiding friction and shear and heels should be lifted free of the bed surface using pillows. The frequency of repositioning should be based on individual patient need relating to their skin tolerance to pressure (e.g. development of areas of blanching hyperaemia) and their general condition and comfort. Pressure should be relieved or redistributed, and repositioning onto bony prominences should be avoided by using the 30-degree tilt options and profiling bed functions. Once patients can sit in a chair, repositioning should be carried out regularly by encouraging patients to stand, mobilise and return to lying positions depending on frequent skin reassessment.

Incontinence: Incontinence of urine and/or faeces exposes the skin to excessive moisture which can damage the dermal and epidermal cells. Urine, faeces, sweat and exudate contain chemical substances which are toxic to skin cells and can lead to incontinence-associated dermatitis [3]. Patients with incontinence should have an 
individual continence management plan that includes immediate cleansing of the skin following incontinence and the light use of barrier creams to protect the skin. The absorbency of continence products such as pads can be affected by barrier creams transferred from the skin to the pad.

Nutrition: Nutritional assessment and screening should be conducted to identify patients who are malnourished or at risk of malnourishment. It is essential to ensure that there is an adequate supply of nutrients-particularly protein, energy, water and vitamins - to the skin. An individualised nutrition plan is needed for anyone with or at risk of malnutrition (see Chap. 9 for further information).

\subsection{Wound Management}

Because the definitive management of hip fracture, and some other fragility fractures that require surgical fixation, almost always involves surgery (see Chap. 5), most patients require acute wound care during the hospital stay and following discharge. Surgical wounds are an important source of potential complications due to risk of infection, haematoma and wound healing problems such as dehiscence.

Surgical wounds occur under controlled circumstances and surgeons endeavour to ensure minimal tissue loss and good approximation of the wound edges during wound closure. The main aim of care of the wound is that it should heal rapidly without complications such as infection or dehiscence (breakdown). However, for many patients who have surgery following fragility fracture, their general health is poor and they have multiple pre-existing health problems and medications which can significantly affect healing. It has been reported that occurrence of deep infection following surgery for hip fracture is between $1.5 \%$ and $7.3 \%$ depending on comorbidities [12]. Understanding the factors that can lead to poor healing and, particularly, surgical site infection as well as the best methods to facilitate healing and prevent infection are important nursing activities both in the pre- and postoperative period as, even preoperatively, a well-prepared patient can make a significant difference to avoiding surgical complications and their consequences.

Wound healing is the process by which function to damaged tissue is restored following surgery. It is a dynamic, complex process that is significantly affected by the nature of the wound, pre- and post-operative management, the patient's health status, the care environment and the care given. Some surgical wounds may be considered a straightforward interruption in the continuity of the protection by the skin resulting from surgery that can be expected to make rapid and predictable progress towards healing [13]. For the older person who has undergone surgery, however, there are numerous factors which place the wound at greater risk of wound healing problems such as infection, haematoma and dehiscence as well as sepsis and death. To facilitate optimal wound healing, the general health and well-being of the patient must be optimised both pre- and post-operatively while considering the patient's past medical/surgical history, medications/polypharmacy and current health history as discussed in Chap. 4. Good nutrition (see Chap. 8) is also central to ensuring wound healing without complications especially given the prevalence of 
malnutrition in elderly hospitalised patients who may have undergone lengthy periods of fasting preoperatively.

\subsubsection{Preventing Wound Infection}

Orthopaedic surgery results in a wound that penetrates through all layers of soft tissue to bones and joints making infection a significant worry as deep surgical site infection can lead to implant site infection (where there has been a need for surgical fracture fixation or hemi- or total arthroplasty), osteomyelitis and wound dehiscence, resulting in pain and discomfort, poor outcomes from surgery and delayed discharge.

The use of the most recent evidence-based guidelines [14] for preventing hospital-acquired infections (HAIs) is central to the prevention of surgical site infection. Such guidelines tend to focus on the following important interventions which should be employed for all patients following fragility fracture and surgery:

- Careful attention to hand hygiene

- Hospital environmental hygiene

Specific measures for the prevention of surgical site infection should also be employed [13] including:

- Careful preoperative preparation and perioperative care including skin preparation and antibiotic prophylaxis according to national and international evidencebased guidance and medical team instructions

- Ensuring the patient's general health status and tissue perfusion is optimised through good nutrition and hydration

- Stringent aseptic technique when caring for wounds and removing and handling wound drains

- Removing wound drains as soon as possible, if possible within $24 \mathrm{~h}$ of surgery

- Covering wounds with an appropriate sterile dressing until it is evident that the initial stages of wound healing have been completed and the wound surface is, at least, superficially sealed

- Removing the dressing and disturbing the wound as little as possible; dressings should only be removed and replaced if there are signs that there has been "strike through" (blood or exudate has soaked through the dressing) or the wound needs inspection because of pain and other symptoms of infection

- Where wound closure materials (e.g. sutures or staples) need removal, this should be done at the appropriate time (when healing is anticipated) and only following careful inspection of the wound; wound closure materials should not be left in for longer than necessary

- Any identified problems with the wound should be reported immediately to the medical team; immediate medical attention is needed if infection is suspected and appropriate antibiotics should then be prescribed 
- Assessment and surveillance of the wound in the post-operative period for signs of infection (wound breakdown (dehiscence) pain, particularly that which is increasing, redness and wound discharge) until recovery is complete; in wounds that involve orthopaedic implants, infection may appear any time up to 1 year after surgery

- Laboratory analysis of wound samples such as swabs can be useful in providing information about what organisms may be colonising the surface of the skin but is not helpful in diagnosing deep infection unless there is wound discharge. Hence, wound samples should only be taken of discharging exudate.

\subsection{Summary of Key Points for Learning}

An understanding of the pathophysiology of pressure injuries by nurses is a fundamental aspect of pressure injury prevention. Nurses need to be able to recognise the risk factors for pressure injury, including red skin, particularly in patients who are at elevated risk, such as those with hip fracture. Evidence-based care should include a focus on skin assessment, support surfaces, keeping the patient moving by ensuring mobility and/or frequent changes of position, ensuring good nutrition and hydration and effectively managing skin moisture, especially relating to incontinence.

The effective evidence-based management of surgical wounds following surgery after fragility fracture can be challenging as ageing and comorbidities affect wound healing. Wound care involves careful wound assessment and observation and attention to infection prevention measures while managing the factors that affect wound healing for individual patients.

\subsection{Suggested Further Study}

- Download the National Pressure Ulcer Advisory Panel, European Pressure Ulcer Advisory Panel and Pan Pacific Pressure Injury Alliance (NPUAP/EPUAP/PPPIA) (2014) Prevention and Treatment of Pressure Ulcers: Quick Reference Guide. From: http://www.epuap.org/wp-content/uploads/2016/10/quick-reference-guidedigital-npuap-epuap-pppia-jan2016.pdf. Identify someone in your team who might be a good person to act as a champion for ensuring the guidelines are implemented in your clinical area (this might, of course, be you or you can support them). Discuss with them which aspects of the guidelines your unit achieves least well or that you feel are the most important to tackle. Discuss how you might approach changing one area of practice to ensure you meet the guidelines.

- Find out where you can access data about wound infection rates (1) nationally and (2) in your unit. How does your unit compare to the national rates? Think about how surgical wound care is practised in your unit compared to the recommendations presented in this chapter. Write a reflection that includes recommendations for at least one improvement in practice and develop an action plan. 


\section{References}

1. Gunningberg L et al (2013) Pressure ulcer knowledge of registered nurses, assistant nurses and student nurses: a descriptive, comparative multicentre study in Sweden. Int Wound J 12:462. https://doi.org/10.1111/iwj.12138

2. NICE (2014) Pressure ulcers: prevention and management. Clinical guideline, vol 179. www. nice.org.uk/guidance/cg179

3. Beeckman D (2017) A decade of research on incontinence-associated dermatitis (IAD): evidence, knowledge gaps and next steps. J Tissue Viability 26:47-56

4. National Pressure Ulcer Advisory Panel, European Pressure Ulcer Advisory Panel and Pan Pacific Pressure Injury Alliance (NPUAP/EPUAP/PPPIA) (2014) Prevention and Treatment of Pressure Ulcers: Quick Reference Guide. In: Haesler E (ed) Cambridge Media: Osborne Park, Australia. http://www.epuap.org/wp-content/uploads/2016/10/quick-reference-guide-digitalnpuap-epuap-pppia-jan2016.pdf

5. Smith I et al (2017) Exploring the role of pain as an early predictor of pressure ulcers: a prospective cohort study. BMJ Open 7:e013623. https://doi.org/10.1136/bmjopen-2016-013623

6. Hommel A et al (2017) Successful factors to prevent pressure ulcers-an interview study. J Clin Nurs 26:182-189. https://doi.org/10.1111/jocn.13465

7. Moore Z, Cowman S (2014) Risk assessment tools for the prevention of pressure ulcers. Cochrane Database Syst Rev (2):CD006471. https://doi.org/10.1002/14651858.CD006471.pub3

8. Sving E et al (2014) Factors contributing to evidence-based pressure ulcer prevention. A crosssectional study. Int J Nurs Stud 51:717-725

9. Vanderwee K et al (2007) Pressure ulcer prevalence in Europe: a pilot study. J Eval Clin Pract 13(2):227-235

10. NHS (2016) Stop the pressure. Helping to prevent pressure ulcers. NHS Improvement. http:// nhs.stopthepressure.co.uk/

11. McInnes E et al (2011) Support surfaces for pressure ulcer prevention. Cochrane Database Syst Rev (4):CD001735. https://doi.org/10.1002/14651858.CD001735.pub4

12. de Jong $L$ et al (2017) Factors affecting the rate of surgical site infection in patients after hemiarthroplasty of the hip following a fracture of the neck of the femur. Bone Joint J 99-B:10881094. https://doi.org/10.1302/0301-620X.99B8

13. Donnelly J et al (2014) Wound management, tissue viability and infection. In: Clarke S, SantyTomlinson J (eds) Orthopaedic and Trauma Nursing. An evidence based approach. Wiley/ Blackwell, Oxford

14. Loveday $\mathrm{H}$ et al (2014) epic3: National evidence-based guidelines for preventing healthcare associated infections in NHS hospitals in England. J Hosp Infect 86(Suppl 1):S1-S70. https:// doi.org/10.1016/S0195-6701(13)60012-2

15. Coleman S et al (2014) A new pressure ulcer conceptual framework. J Adv Nurs 70(10):2222-2234

Open Access This chapter is licensed under the terms of the Creative Commons Attribution 4.0 International License (http://creativecommons.org/licenses/by/4.0/), which permits use, sharing, adaptation, distribution and reproduction in any medium or format, as long as you give appropriate credit to the original author(s) and the source, provide a link to the Creative Commons license and indicate if changes were made.

The images or other third party material in this chapter are included in the chapter's Creative Commons license, unless indicated otherwise in a credit line to the material. If material is not included in the chapter's Creative Commons license and your intended use is not permitted by statutory regulation or exceeds the permitted use, you will need to obtain permission directly from the copyright holder. 